\title{
A FINITE ELEMENT FORMULATION OF THE LARGE SLIDING CONTACT
}

\author{
G. J. M. A. SCHREPPERS, W. A. M. BREKELMANS AND A. A. H. J. SAUREN \\ Department of Mechanical Engineering, Eindhoven University of Technology, Eindhoven, The Netherlands
}

\begin{abstract}
SUMMARY
A finite element formulation for the large sliding contact problem is discussed. With respect to a set of two material points the free and contacting state are distinguished. Because of the possibly occurring large slidings, the two points contact conditions are transformed to contact conditions with respect to larger sets of points, the so-called contact units. An incremental solution strategy is presented which calculates the equilibrium solution that obeys these contact conditions using an iterative procedure. Part of this strategy is the identification of actual sets of contacting material points. The performance of the algorithm is demonstrated by some case-studies.
\end{abstract}

\section{INTRODUCTION}

The finite element method is a powerful tool for the analysis of mechanical structures with complex geometries. Nowadays, there is a strong interest in applications of the finite element method to non-linear problems. Three kinds of non-linearities can be distinguished.

(i) geometrical non-linearities, as a result of large displacements,

(ii) physical non-linearities, stemming from non-linear material behaviour, and

(iii) constraint non-linearities, caused by changing contact interaction.

In this paper a finite element formulation for static contact problems is presented, which is focussed on large sliding movements of contacting bodies. The proposed algorithm is well comparable with the state of the art methods as described in the literature (e.g. Reference 6). However, to attain a more efficient solution process, special attention is paid to the formulation of the conditions for contact-state transitions. Frictional behaviour is left out of consideration.

In the literature (e.g. Hughes et al., ${ }^{5}$ Bathe and Chaudhary ${ }^{2}$ ) the contact of two material points and the appropriate constraints is often discussed. Standard procedures are available to solve constrained problems such as the Lagrange multiplier method and the penalty function formulation. Guerra and Browning, ${ }^{4}$ comparing these formulations, showed that the Lagrange multiplier method satisfies the contact constraints more precisely. This method, however, adds extra degrees of freedom requiring additional storage and computational effort. Further, they noticed that in most cases problems were encountered with respect to the convergence of the solution process.

It is the experience of the authors of the present paper that the ambiguity of the criteria to identify sets of actual contacting material points constitutes an important aspect of the contact algorithm. This is especially the case when large slidings in the contact areas occur. Till now relatively little attention has been paid to this point in literature. Recently, Zhong and Nilsson ${ }^{8}$ described a contact searching algorithm and specified contact criteria for sets of material points. 
However, for large sliding contacts, the actual composition of the sets of contacting points changes continuously even as the corresponding constraints. No special attention was given by Zhong and Nilsson ${ }^{8}$ to these aspects.

After the contact behaviour has been specified, a general formulation of the discretized problem is given and the solution strategy is outlined. Next, the possible contact states of a material point in the discrete formulation and the modelling of the state transitions are discussed. Finally, the performance of the algorithm is illustrated and evaluated by some examples.

\section{CONTINUOUS FORMULATION OF CONTACT BEHAVIOUR}

Consider a system consisting of one or more bodies. A material point $\mathrm{P}$ on the outer surface is identified by its material surface co-ordinates

$$
\xi^{\mathbf{P}}=\left[\begin{array}{l}
\xi_{1}^{\mathbf{P}} \\
\xi_{2}^{\mathbf{P}}
\end{array}\right]
$$

The total outer surface, that comprises all material points on the boundaries of the bodies in the system, is indicated by $A$. It is supposed that this surface is smooth, such that the normal direction is uniquely defined. The volume of the bodies of the system is denoted by $V$.

With respect to an arbitrary material point $\mathrm{P}$ of $A$, two contact states can be distinguished:

1. At time $\tau=t$ point $\mathrm{P}$ is contacting another material point of $A$. This is the case if a point $\mathrm{Q}$ on $A$ exists, for which the position vector at time $\tau=t$ is equal to the position vector of $\mathrm{P}$

$$
\mathbf{x}\left(\xi^{\mathbf{P}}, t\right)=\mathbf{x}\left(\xi^{\mathbf{Q}}, t\right)
$$

while at the same time the unit outward normal vectors to the outer surface at $\mathrm{P}$ and $\mathrm{Q}$ are opposite

$$
\mathbf{n}\left(\xi^{\mathbf{P}}, t\right)=-\mathbf{n}\left(\xi^{\mathrm{Q}}, t\right)
$$

Then the contacting state with respect to the point $\mathrm{Q}$ is attributed to $\mathrm{P}$. The principle of action and reaction has to be satisfied

$$
\mathbf{p}_{\mathbf{c}}\left(\xi^{\mathbf{P}}, t\right)=-\mathbf{p}_{\mathbf{c}}\left(\xi^{\mathrm{Q}}, t\right)
$$

$\mathbf{p}_{\mathbf{c}}$ being the contact stress vector. Because no friction is assumed in the interaction, the tangential component of the contact stress vector has to be zero. This condition is represented by

$$
\mathbf{p}_{\mathbf{c}}\left(\xi^{\mathbf{P}}, t\right) \cdot\left[\mathbf{I}-\mathbf{n}\left(\xi^{\mathbf{P}}, t\right) \mathbf{n}\left(\xi^{\mathbf{P}}, t\right)\right]=\mathbf{0}
$$

which describes a constitutive relationship for the contacting state. An additional constitutive relationship results from the condition that the contact stress has to be compressive

$$
\mathbf{P}_{\mathbf{c}}\left(\xi^{\mathbf{P}}, t\right) \cdot \mathbf{n}\left(\xi^{\mathbf{P}}, t\right) \leqslant 0
$$

2. If point $\mathrm{P}$ is not coinciding with any other material point of $V$ at time $\tau=t$, the free state is attributed to $P$. Characteristic for the constitutive behaviour of the free state is the equation

$$
\mathbf{p}_{\mathrm{c}}\left(\xi^{\mathbf{P}}, t\right)=\mathbf{0}
$$

Penetration of point $\mathrm{P}$ into a body of the system is not allowed.

\section{DISCRETIZATION}

The total outer surface of the system under consideration is decomposed according to

$$
A=A_{\mathrm{c}}+A_{\mathrm{n}}
$$


where $A_{\mathrm{c}}$ is a fixed set of material points, which are possibly interacting with other points of $A_{\mathrm{c}}$, and $A_{\mathrm{n}}$ is the complement of $A$ and $A_{\mathrm{c}}$. Points of $A_{\mathrm{c}}$ can be free or contacting. Assuming body forces to be negligible, the weak formulation of equilibrium reads

$$
\int_{V}(\nabla \mathbf{w})^{\mathrm{c}}: \sigma \mathrm{d} V-\int_{A_{\mathrm{n}}} \mathbf{w} \cdot \mathbf{p} \mathrm{d} A-\int_{A_{\mathrm{c}}} \mathbf{w} \cdot \mathbf{p}_{\mathrm{c}} \mathrm{d} A=0
$$

where $\sigma$ is the Cauchy stress tensor, $w$ is an arbitrary vector weighting function and $\nabla$ the gradient operator with respect to the actual configuration. The load on $A_{\mathrm{n}}$ is represented by the stress vector $\mathbf{p}$.

For the field of actual position vectors, a finite element discretization is introduced according to

$$
\mathbf{x}=\underline{\Psi}^{\mathbf{T}} \underline{x}
$$

$\underline{x}$ being the column of nodal co-ordinates with respect to an appropriate vector base, and $\Psi$ being the column with vector interpolation functions expressed in material co-ordinates. Using a Galerkin approach for the weighting function

$$
\mathbf{w}=\underline{\Psi}^{\mathrm{T}} \underline{w}
$$

with the column $w$ defined analogous to $\underset{x}{x}$, it can be derived from equation (9) that

$$
\underline{w}^{\mathrm{T}}(\mathfrak{f}-\underset{r}{r}-\tilde{c})=0
$$

The columns $f, \underline{r}$ and $\underset{c}{c}$ contain the internal nodal forces, the external nodal forces applied on $A_{\mathrm{n}}$ and the contact forces on $A_{c}$, respectively. These columns are defined by the equations

$$
\begin{aligned}
& f=\int_{V}(\nabla \Psi)^{c}: \sigma \mathrm{d} V \\
& \underline{L}=\int_{A_{\mathrm{n}}} \underset{\psi}{\Psi} \cdot \mathrm{p} \mathrm{d} A \\
& \mathcal{c}=\int_{A_{\mathrm{c}}} \Psi \cdot \mathbf{p}_{\mathrm{c}} \mathrm{d} A
\end{aligned}
$$

It is supposed that the column of internal nodal forces can be expressed in the nodal co-ordinates

$$
\mathcal{L}=\mathfrak{L}(\underset{\sim}{x})
$$

The column $r$ satisfies the user-supplied dynamic boundary conditions. Corresponding components of $r$ and $\mathcal{c}$ cannot both be unequal to zero.

The requirement that equation (12) applies all arbitrary sets $w$ results in the balance of nodal forces

$$
f-\underline{r}-\underline{c}=\underline{0}
$$

The number of equations in (17) is defined to be $3 m, m$ being equal to the total number of nodes in $V$. The maximum number of non-trivial components in $€$ equals to $3 n, n$ being the number of nodes on $A_{\mathrm{c}}$.

Besides the equilibrium equations, also the contact conditions should be satisfied. The contact situation is defined by the assigned contact states of the points of $A_{c}$, including the partner points in case of the contacting state. Because of history dependence of the problem, caused by changing contact situations, the load is applied incrementally. The discrete contact problem is then formulated by the discretized equilibrium equations (17) with respect to the end of an increment along with the appropriate contact conditions for all nodes of $A_{c}$. These conditions are elaborated in the next section. 


\section{SOLUTION STRATEGY}

While in the continuous formulation for the contacting state attention has been focussed on a particular material point $\mathrm{P}$ on $A_{\mathrm{c}}$, in contact with another material point $\mathrm{Q}$ on $A_{\mathrm{c}}$, in the discrete formulation a nodal point $\mathrm{P}$ on $A_{\mathrm{c}}$ is considered to interact with several other nodes on $A_{\mathrm{c}}$. For an adequate description the contact unit is introduced. A contact unit is defined with respect to any node $\mathrm{P}$ on $A_{\mathrm{c}}$ and consists of node $\mathrm{P}$ and a set of nodes in the part of $A_{\mathrm{c}}$ that may interact with node $\mathrm{P}$. In general one node will participate in more than one contact unit.

Suppose that with respect to every node on $A_{\mathfrak{c}}$ a contact unit is defined. The column $\mathcal{E}$ is composed of the nodal interaction contributions of all the contact units in the system. Let $k_{\mathrm{p}}(3$ components) be the column with the the nodal force contributions on node $P$ as a result of the interaction in the particular contact unit defined for $P$.

It is assumed that to every contact unit one of the following two contact states has been assigned (the procedure to assign a certain contact state will be explained later on).

\section{Passive state}

This state corresponds to the situation previously called free. According to equation (7) the contact behaviour for the contact unit is characterized by

$$
k_{\mathrm{p}}=0
$$

\section{Active state}

This state is the equivalent of the contacting state of a point in the continuous formulation. Because $k_{\mathrm{p}}$ is the force contribution on node $\mathrm{P}$ of the contact unit, $-k_{\mathrm{p}}$ is the action of node $\mathrm{P}$ on a number of other nodes in this contact unit, according to the principle of action and reaction. The distribution of $-k_{\mathfrak{p}}$ over these nodes is formally given by

$$
\Delta_{\mathrm{p}} \mathcal{E}=-\underline{D}_{\mathrm{p}}(x) k_{\mathrm{p}}
$$

where $\underline{D}_{\mathrm{p}}$ ( $3 m$ rows, 3 columns) is a distribution matrix, dependent on the geometry at the end of the increment as defined by the column $x$ with nodal co-ordinates. Then the total contribution to $\mathcal{c}$ of the contact unit under consideration is represented by

$$
\mathfrak{E}_{\mathrm{p}}=\Delta_{\mathrm{p}} \mathfrak{\epsilon}+\underline{L}_{\mathrm{p}} \underline{k}_{\mathrm{p}}=\left[-\underline{D}_{\mathrm{p}}(x)+\underline{L}_{\mathrm{p}}\right] \underline{k}_{\mathrm{p}}
$$

with $\underline{L}_{\mathrm{p}}$ ( $3 m$ rows, 3 columns) being the appropriate location matrix. Because of equation (5) the tangential contact forces are prescribed to be zero. The corresponding conditions with respect to $k_{\mathrm{p}}$ can be written as

$$
\underline{P}_{\mathrm{p}}(\underline{x}) k_{\mathrm{p}}=0
$$

with $\underline{P}_{\mathrm{p}}$ ( 2 rows, 3 columns) the projection matrix to be derived from the actual geometry.

Finally, a kinematic condition applies for node $\mathrm{P}$, taking into account that the contact unit is closed, which means that node $\mathrm{P}$ is localized in a particular element face of $A_{\mathrm{c}}$. This can be formulated by

$$
\kappa_{\mathrm{p}}(x)=0
$$

where $\kappa_{\mathrm{p}}$ is a scalar function of the nodal co-ordinates.

The equations (21) and (22) are denoted in an explicit form. However, only for linear interpolation functions on element-level can an explicit expression be formulated. For higher order elements this is not possible. Because in the following only derivatives of these conditions and the values of the left hand sides of (21) and (22) are used, this is not a serious problem. 
The set of contact units to which the active state has been assigned is denoted by $S$, the number of active units by $s$. Summation over all the active contact units in the system yields

$$
\mathcal{E}=\sum_{\mathbf{p} \in S} \mathcal{C}_{\mathfrak{p}}=\sum_{\mathbf{p} \in S}\left[-\underline{D}_{\mathbf{p}}(\underline{x})+\underline{L}_{\mathfrak{p}}\right] \underline{k}_{\mathfrak{p}}=\underline{A}(\underline{x}) \underline{k}
$$

with the matrix $\underline{A}$ ( $3 m$ rows, $3 n$ columns) controlling the assemblage and $\underline{k}$ ( $3 n$ components) the column with nodal force contributions $\underline{x}_{\mathrm{p}}$ of all the contact units. The conditions (18), (21) and (22) are similarly denoted by

$$
\begin{array}{r}
\underline{Z k}=\underline{0} \\
\underline{P}(\underline{x}) \underline{\underline{k}}=\underline{0} \\
\underline{\underline{k}}(\underline{x})=\underline{0}
\end{array}
$$

respectively, with $\underline{Z}(3(n-s)$ rows, $3 n$ columns), $\underline{P}$ ( $2 s$ rows, $3 n$ columns) and $\underline{\kappa}$ (s components) implicitly defined. The balance equation (17) and the conditions (24), (25) and (26) constitute a set of $3(m+n)$ equations, describing the discrete contact problem. In a more concise notation these can be written as

$$
\left.\begin{array}{rl}
f(x)-r-\mathcal{c}(\underline{x}, \underline{k}) & =\underset{0}{0} \\
\underline{h}(\underline{x}, \underline{k}) & =\tilde{0}
\end{array}\right\}
$$

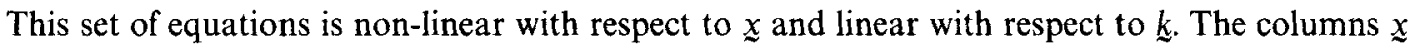
and $k$ contain the system degrees of freedom.

Because the problem formulation is essentially non-linear, an iterative solution procedure is used to calculate the system degrees of freedom at the end of each increment, starting from estimates according to the converged situation of the preceding increment. Modifications of the estimates for the system degrees of freedom are indicated by $\delta \underset{x}{ }$ and $\delta \underline{k}$, corresponding modifications of the estimates for $f, \underset{c}{c}$ and $\underline{h}$ by $\delta f \underset{\sim}{f} \delta \underline{\sim}$ and $\delta \underline{h}$, respectively. Linearization with respect to $\underline{x}$ and $k$ yields the relationships

$$
\begin{aligned}
{ }^{i+1} \delta \underline{f} & ={ }^{i} \underline{F}_{x}{ }^{i+1} \delta \underline{x} \\
{ }^{i+1} \delta \underline{c} & ={ }^{i} \underline{C}_{x}{ }^{i+1} \delta \underline{x}+{ }^{i} \underline{C}_{k}{ }^{i+1} \delta \underline{\underline{k}} \\
{ }^{i+1} \delta \underline{h} & ={ }^{i} \underline{H}_{x}{ }^{i+1} \delta \underline{x}+{ }^{i} \underline{H}_{k}{ }^{i+1} \delta \underline{\underline{k}}
\end{aligned}
$$

with the left upper indices referring to the iteration number $(i=1,2, \ldots)$. The matrices ${ }^{i} \underline{F}_{x},{ }^{i} \underline{C}_{x}$, ${ }^{i} \underline{C}_{k},{ }^{i} \underline{H}_{x}$ and ${ }^{i} \underline{H}_{k}$ are the derivatives of $f, \underline{c}$ and $\underline{h}$ with respect to $\underline{x}$ and $\underline{k}$ for the $i$ th estimates ${ }^{i} \underline{x}$ and $i k$ of the actual increment. Based on (2) the following equations to determine approximations for $\delta \underset{\sim}{x}$ and $\delta \underline{k}$ are obtained:

$$
\left[\begin{array}{ll}
{ }^{i} \underline{F}_{x}-{ }^{i} \underline{C}_{x} & -{ }^{i} \underline{C}_{k} \\
{ }^{i} \underline{H}_{x} & { }^{i} \underline{H}_{k}
\end{array}\right]\left[\begin{array}{l}
\delta \underline{x} \\
\delta \underline{k}
\end{array}\right]=\left[\begin{array}{l}
-{ }^{i} \tilde{f}+\underline{r}+{ }^{i} \mathcal{c} \\
-{ }^{i} \underline{h}
\end{array}\right]
$$

Although the conditions (24) and (25), requiring linear combinations of components of $\underline{k}$ to be zero, have not necessarily to be taken into account, they are considered in the present formulation. In this way a set of equations is obtained, the number of which is independent of the actual contact states. When the conditions (24) and (25) are not taken into account in (31), the bottom right hand sub-matrix ${ }^{i} \underline{H}_{k}$ is zero (e.g. Bathe and Chaudhary ${ }^{2}$ ).

The columns ${ }^{i} \underset{x}{x}$ and ${ }^{i} k$ are updated according to

$$
{ }^{i+1} \underline{x}={ }^{i} \underline{x}+{ }^{i+1} \delta \underline{x}
$$




$$
{ }^{i+1} \underline{k}=\underline{i} \underline{\underline{k}}+{ }^{i+1} \delta \underline{k}
$$

and the iteration process is continued until convergence is reached. With respect to the convergence criterion, not only the right hand side of equation (31) (the residual) has to be negligibly small, but also the inequality conditions relating to prevention of penetration as well as to maintenance of the compressive character of interaction forces have to be satisfied. These conditions are not covered by (27) and they will often require changes of the initially assigned contact state. For computational reasons the contact state is allowed to change every iteration cycle. To achieve convergence of the iteration process contact-state transitions, which will be specified in the next section, play an important role.

\section{CONTACT-STATE TRANSITIONS}

In this section the state transitions for the present algorithm are outlined. These transitions are derived from the actual estimates for the columns $\underset{\sim}{x}$ and $\underline{k}$ for every iteration again. On the basis of these estimates it is determined whether for a contact unit the passive or active state will be attributed and in case of the active state also the element face is established with which the unit node $\mathrm{P}$ is in contact. It is assumed that all units have the passive state at the start of an analysis.

\section{Transition from the passive state}

If after iteration $i$ the passive state has been attributed to the contact unit with respect to node $\mathrm{P}$ there is no mathematical restriction to node $\mathrm{P}$ against penetrating $A_{\mathrm{c}}$. After iteration $i+1$ it has to be checked whether this node passed any of the element faces of the contact unit. Therefore, the trajectories of the nodal points

$$
\underline{x}(\mu)={ }^{1} \underline{x}+\mu\left({ }^{i+1} \underline{x}-{ }^{1} \underline{x}\right), \quad 0 \leqslant \mu \leqslant 1
$$

from the beginning of the increment to the actual estimate are considered. All values of $\mu$ are calculated for which node $\mathrm{P}$ is located exactly on an element face of the contact unit. The way $\mu$ is calculated depends on the order of the elements on the contact boundaries. For linear interpolation functions $\mu$ can be solved directly from a quadratic equation. For higher order interpolation functions a non-linear system of equations in the material surface co-ordinates has to be solved using standard algorithms.

Two cases are distinguished:

(i) Such a $\mu$ does not exist, which means that node $\mathrm{P}$ did not penetrate any of the element faces of the contact unit and thus the passive state can be maintained.

(ii) There are one or more values of $\mu$ for which $\mathrm{P}$ is located on an element face. Then the smallest value of $\mu$ is considered. For iteration $i+1$ the active state with respect to the related element face is attributed in such a way that the penetration with respect to this element face is eliminated by condition (22).

\section{Transition from the active state}

In the active state node $P$ is allowed to slip without friction over an element face. Starting from the active state in interation $i$ the following strategy is proposed:

(i) When the components of $\underline{k}_{\mathrm{p}}$ do not represent a compressive contact force and so are in contradiction to (6), the passive state is attributed.

(ii) In all other cases (compressive contact force) continuation of the active state is assumed. According to this state the kinematical condition (22) is applied requiring node $P$ to be 
coplanar to the element face to which it is coupled. If in iteration $i+1$ node $\mathrm{P}$ is situated on the same element face as in iteration $i$, $\mathrm{P}$ is coupled to this face. However, if node $\mathrm{P}$ left this element face during the iteration $i+1$, it is automatically coupled to the adjacent element face. The sliding movement from one element face to an other is controlled in this way.

\section{EXAMPLES}

To demonstrate the performance of the contact algorithm, some case-studies are discussed in the following. Because the contact algorithm is essentially the same for three- and two-dimensional analyses, for reasons of simplicity only plane and axisymmetric examples will be presented. Consequently $A_{\mathrm{c}}$ is defined by line segments bounded by two nodes.

In the first example the process of coming into contact and the breaking of contact is simulated by moving a small elastic square element in the direction of a spatially fixed rigid large square element in the first increment and pulling them apart in the second increment. Figure 1(a) shows the reference configuration, in which the prescribed displacements are indicated by vectors. Because the passive state is attributed to all nodes in the reference configuration, only rigid body displacements occur in the first iteration. At the end of this step (Figure 1(b)) two nodes of the small square have penetrated an element face of the large square and the penetrations are calculated. In the next iteration the active state is assigned to these nodes and penetrations are corrected as is shown in Figure 1(c), while equilibrium is also achieved.

In the second increment the bodies are pulled apart by prescribing displacements as indicated by the vectors in Figure 1(d). Because at first the active state is continued, contact is maintained and pulling contact forces will occur (Figure 1(e)). Then the passive state takes over for the next iteration and the final situation with zero stresses is given in Figure 1(f).

In the second example the corner contact problem shown in Figure 2(a) is examined. Baaijens ${ }^{1}$ mentioned this problem as an illustration of stability aspects in relation to choosing contact nodes and contact element faces. In this example the nodes 5, 6, 8, 9, 10 and 11 are considered to constitute $A_{\mathrm{c}}$. Node 11 is fixed in space. Displacements are prescribed as indicated by the vectors in Figure 2(a). After a rigid body displacement step (Figure 2(b)), the penetrations

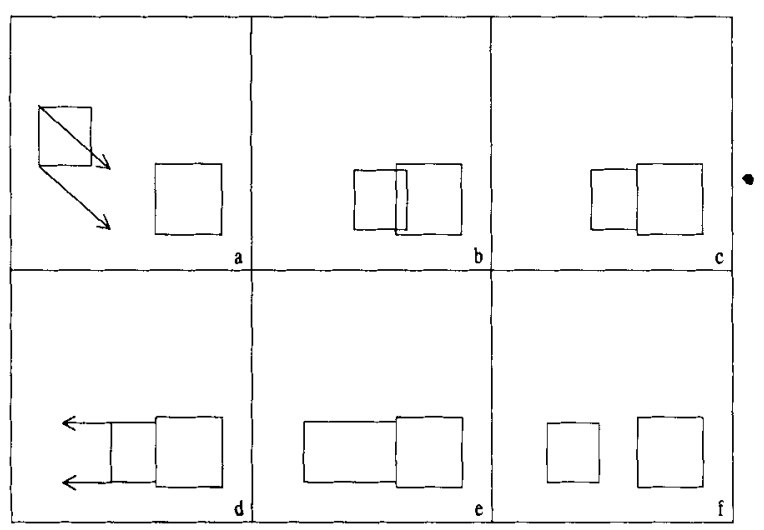

Figure 1. Subsequent iteration estimates 


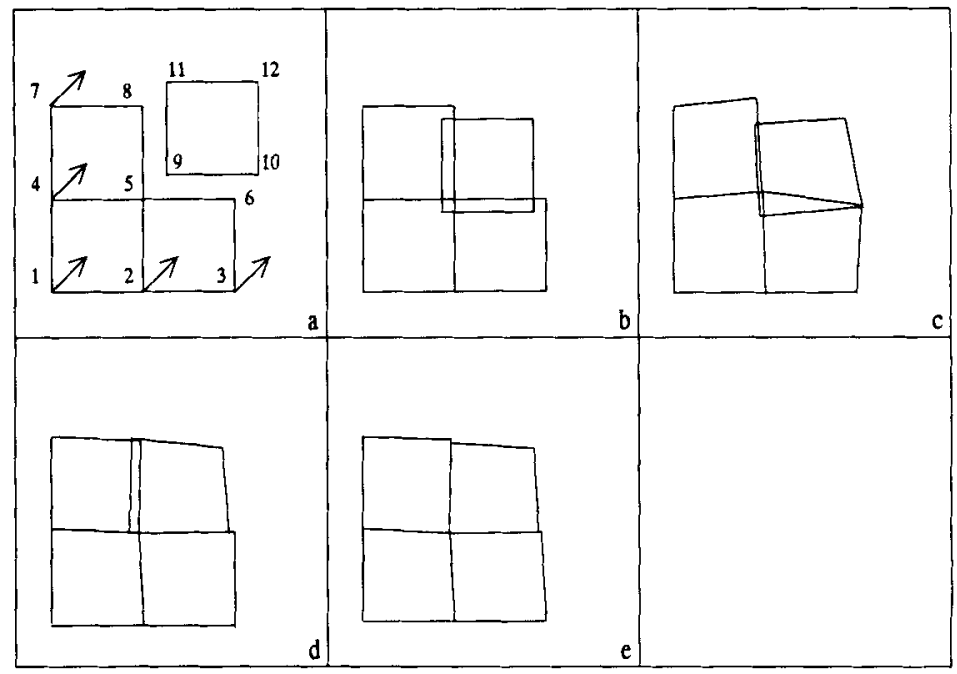

Figure 2. Corner contact problem

are calculated. Then the active state is assumed for a set of nodes according to:

\begin{tabular}{l|c|c|c|c|c|c} 
node & 5 & 6 & 8 & 9 & 10 & 11 \\
\hline in contact with element face & $9-11$ & $9-10$ & $9-11$ & $5-8$ & $5-6$ & $5-8$
\end{tabular}

Node 5 is arbitrarily coupled to element face $9-11$, alternatively it could be coupled to element face 9-10. Similarly node 9 can be considered contacting either face 5-6 or face 5-8. However, for the present combinations of nodes and faces, the active state constraints (22) for these nodes are dependent because both nodes 5 and 9 are projected to parallel planes. This combination of active state constraints does not prevent node 9 from penetrating element face 5-6. Penetration occurs as shown in Figure 2(c). Now, node 9 slipped off element face 5-8, and in the next iteration step the active state is attributed to node 9 (in contact with face 5-6). A situation occurs in which the coinciding nodes 5 and 9 both have the active state with respect to non-parallel element faces. Constraints applied to coinciding nodes to non-parallel element faces suppress the relative motion of these nodes. In the meanwhile the nodes 8 and 6 move off their respective element faces and finally the equilibrium situation is achieved in Figure 2(e). The situations in Figures 2(c) and 2(d) have to be considered as entr' actes and are avoided when the nodes 5 and 9 are bounded to orthogonal element faces in the second iteration step.

Large slidings are considered in the third example. Figure 3(a) shows a spatially fixed point obstacle represented by node 12 , and ten beam elements between the nodes 1 to 11 . In node 1 the horizontal displacement is prescribed as indicated by the vector, while the vertical displacement and rotation in this point are suppressed. In the fifth increment (Figure (3f)) the beam comes into contact with the obstacle and the upper nine beam elements start bending, while the lower one is still not loaded and makes a rigid body movement. In the following increments the element faces slip over node 12 , node 10 being passed in the 12 th increment. In the 15 th increment the end of the beam has passed the obstacle, and an unstressed equilibrium situation results. 


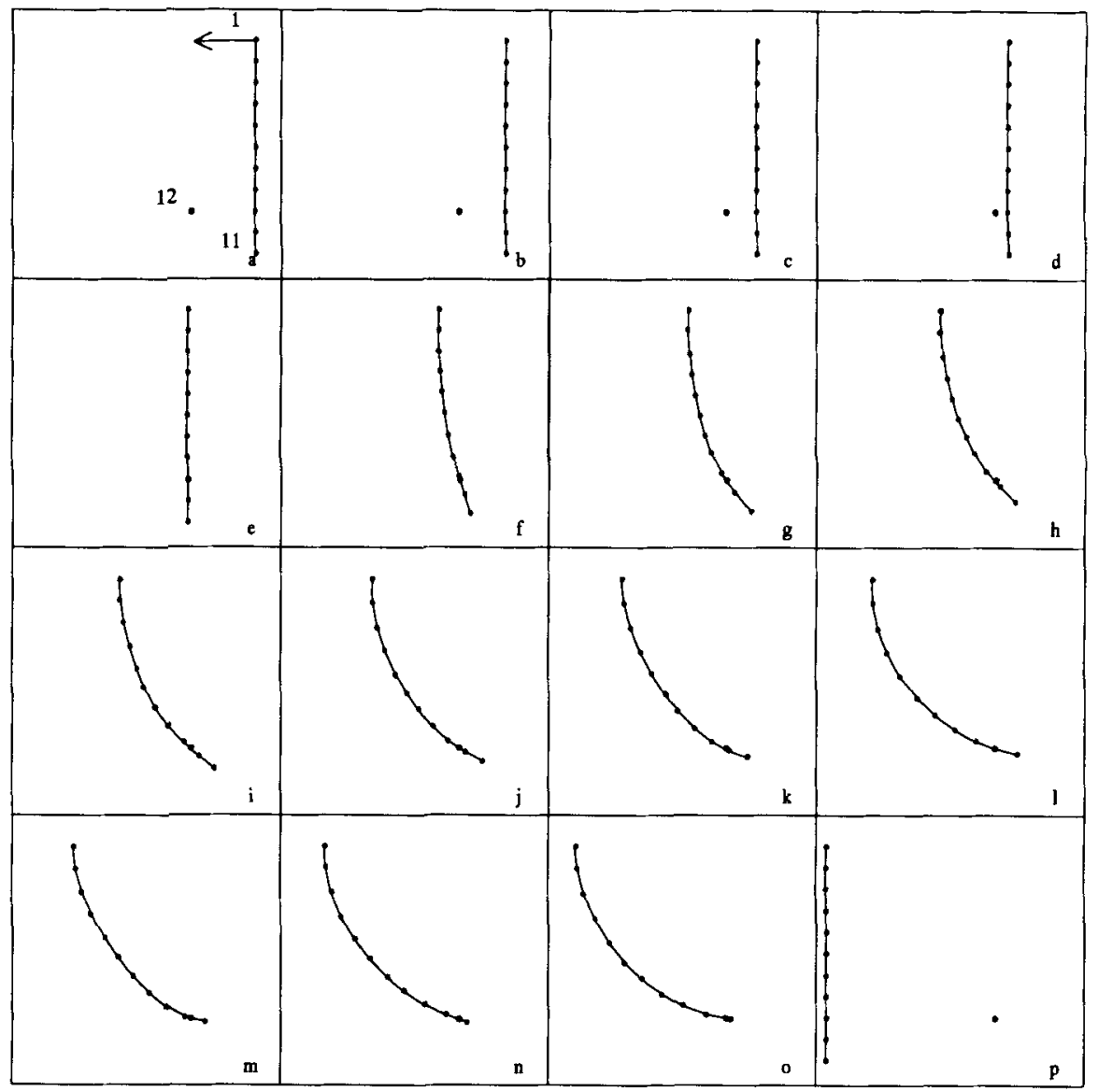

Figure 3. Beam and fixed obstacle in subsequent increments

In the last example an axisymmetric problem consisting of three separate elastic bodies is considered. These bodies constitute a model of the meniscal contact area in the knee joint (Schreppers $e t a .^{7}$ ). Figure 4(a) shows the reference configuration of these three bodies. In the first increment a rigid body movement is prescribed so that the bodies just match, but are still stressfree (Figure 4(b)). Next, an axial compression is applied in such a way that the upper and lower surfaces of the model remain plane and perpendicular to the axis of symmetry. The result is displayed in Figure 4(c).

\section{DISCUSSION}

The examples presented demonstrate various contact problems with possible state transitions. The stability of the algorithm proves to be quite satisfactory for general contact problems.

Contrary to contacts without slip for which the interaction is defined with respect to fixed pairs of material points, for the large sliding contact the identification of sets of material points is not arbitrary because in general several material points can simultaneously be in contact with the same point. The contact unit is characteristic for the presented formulation and is defined for all 


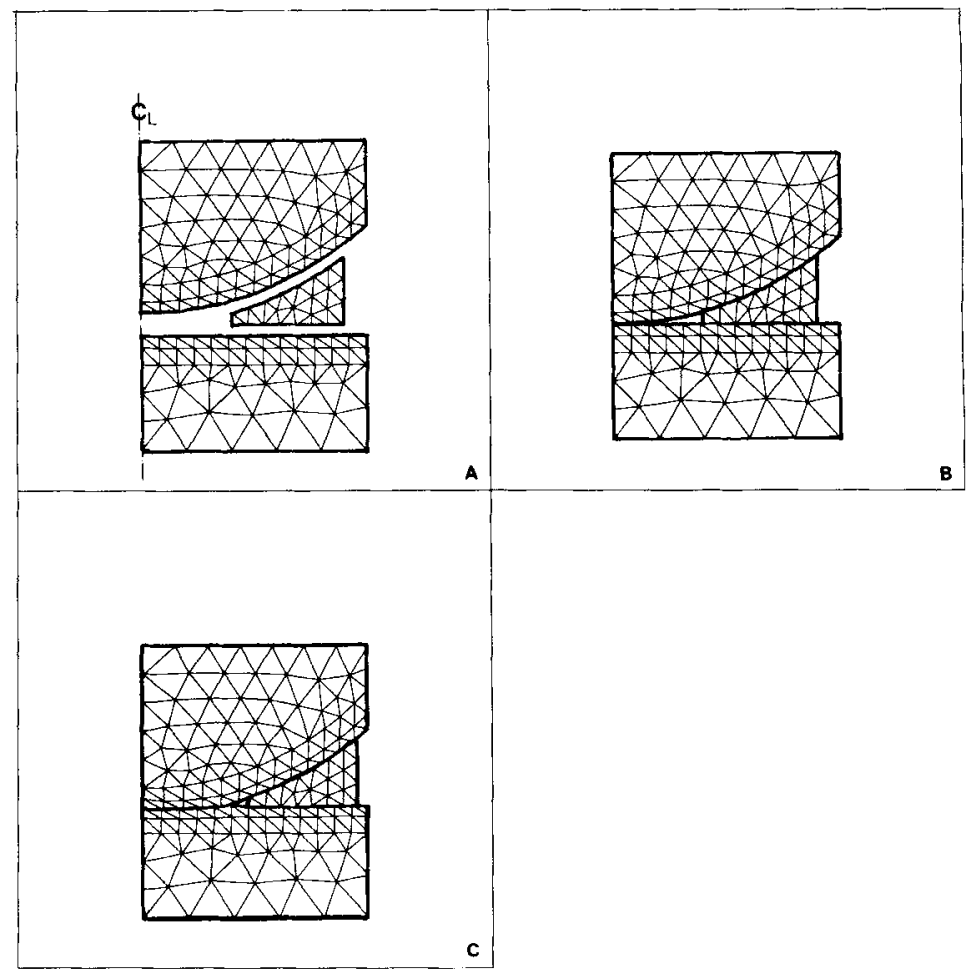

Figure 4. Axisymmetric tibio-femoral joint model

nodes on $A_{\mathrm{c}}$. The state transitions from one element face to an other are controlled by the algorithm in such a way that ambiguity with respect to sets of actual contacting material points is avoided.

In the actual implementation of the present contact formulation all nodes on $A_{\mathrm{c}}$ are considered to constitute one element: the so-called contact element, containing contact units for each of the nodes. To account for the contact constraints and the internal contact forces, only the right hand column and the tangential matrix with respect to this element have to be composed. Such a program structure offers the facility to control the element face transitions, which is characteristic for this algorithm for solving large sliding problems.

Because in the formulation the contact unit is defined for every node on $A_{\mathrm{c}}$ it is not necessary for the user to distinguish between an object body and a target body as is usually done in other formulations. However, because of possible dependences in the system of equations, emanating from the involvement of all nodes on $A_{\mathrm{c}}$, special action has to be taken to solve the equations.

\section{ACKNOWLEDGEMENT}

The authors wish to thank Dr D. G. Roddeman of the TNO Institute of Building Materials and Structures for his extensive contributions to the implementation of the presented algorithm in the TNO-DIANA finite element package. ${ }^{3}$ 


\section{REFERENCES}

1. F. P. T. Baaijens, 'On a numerical method to solve contact problems', Ph.D. Thesis, Eindhoven University of Technology, 1987.

2. K. J. Bathe and A. Chaudhary, 'A solution method for planar and axisymmetric contact problems', Int. $j$. numer. methods eng., 21, 65-88 (1985).

3. DIANA finite element analysis, User Manual, TNO Institute for Building Materials and Structures, Rijswijk, The Netherlands, 1990.

4. F. M. Guerra and R. V. Browning, 'Comparison of two sideline methods using Adina', Comp. Struct., 17, 819-834 (1983).

5. Th. J. R. Hughes, R. L. Taylor, J. L. Sackman, A. Curnier and W. Kanoknukulchai, 'A finite element method for a class of contact-impact problems', Comp. Methods Appl. Mech. Eng., 8, 249-276 (1976).

6. J. Theor. Appl. Mech., Special Issue no. 1, 7, 1-276 (1988).

7. G. J. M. A. Schreppers, A. A. H. J. Sauren and A. Huson, 'A numerical model of the load transmission in the tibiofemoral contact area', J. Eng. Medicine, 204, 53-59 (1990).

8. Z. Zhong and L. Nilsson, 'A contact searching algorithm for general 3-D contact-impact problems', Comp. Struct., 34, $327-335(1990)$. 